\title{
PREDICTIVE MAINTENANCE OF INDUCTION MOTORS IN THE CONTEXT OF INDUSTRY 4.0
}

\author{
Eva Masero Rubio ${ }^{1}$, Rogério Pais Dionísio ${ }^{2}$, Pedro Miguel Baptista Torres ${ }^{3}$ \\ ${ }^{1}$ Allbesmart, Lda, Avenida do Empresário, \\ $n^{\circ}$ 1, 6000-767 Castelo Branco, Portugal \\ ${ }^{2,3}$ Escola Superior de Tecnologia, Instituto Politécnico de Castelo Branco, \\ 6000-767 Castelo Branco, Portugal \\ emasero@allbesmart.pt,rdionisio@ipcb.pt,pedrotorres@ipcb.pt
}

\begin{abstract}
The present paper describes the state of the art related to IIoT Devices and CyberPhysical systems and presents a use case related to predictive maintenance. Industry 4.0 is the boost for smart manufacturing and demands flexibility and adaptability of all devices/machines in the shop floor. The machines must become smart and interact with other machines inside and outside the industries/factories. The predictive maintenance is a key topic in this industrial revolution. The reason is based on the idea that smart machines must be capable to automatically identify and predict possible faults and actuate before they occur. Vibrations can be problematic in electrical motors. For this reason, we address an experimental study associated with an automatic classification procedure,

Based on two distinct architectures to detect anomalies: National Instruments acquisition board and an Arduino board with an EtherCAT Shield. With the implementation of a supervised learning model, both approaches corroborate the applicability and usefulness of this machine learning algorithm to predict vibration faults.
\end{abstract}

Keywords: Cyber-Physical Systems, Industrial IoT, Smart Factories, Machine Learning, Predictive Maintenance.

\section{Introduction}

The Internet of Things (IoT) is a novel paradigm that is rapidly gaining ground in the scenario of modern wireless telecommunications. The basic idea of this concept is the pervasive presence around us of a variety of things or objects - such as RadioFrequency IDentification (RFID) tags, sensors, actuators, mobile phones, etc. - which, through unique addressing schemes, can interact with each other and cooperate with their neighbours to reach common goals [1-2].

Information and communication systems are invisibly embedded in the environment around us. In this generation, there are enormous amounts of data which must be stored, processed and presented in a seamless, efficient and easily interpretable form. Cloud computing can provide the virtual infrastructure for such utility computing which integrates monitoring devices, storage devices, analytics tools, visualization platforms and client delivery [3]. The cost-based model that Cloud computing offers will enable end-to-end service provisioning for businesses and users to access applications on demand from anywhere [4].
While the IoT affects among personal electronic devices, healthcare, or smart homes, the Industrial Internet of Things (IIoT) refers to industrial environments.

The IoT is expected to offer promising solutions to transform the operation and role of many existing industrial systems such as transportation systems and manufacturing systems. E.g., when IoT is used for creating intelligent transportation systems, the transportation authority will be able to track each vehicle's existing location, monitor its movement, and predict its future location and possible road traffic [5].

This document presents the current trends in IIoT research pushed by applications and the need for convergence in several interdisciplinary technologies. Specifically, it presents:

- Overall IIoT vision, cyber-physic systems and some possible platforms to implement it (Section 2)

- Communication Protocols (Section 3)

- OPC Servers (Section 4)

- Use Case using NI LabVIEW software for developing systems and data Acquisition with NI DAQ 6008 and Ethercat (Section 5). 


\section{IIoT and Cyber-Physical Systems}

Nowadays, embedded, mobile, and cyber-physical systems (CPS) are ubiquitous and used in many applications, from industrial control systems, modern vehicles, to critical infrastructure. Current trends and initiatives, such as Industry 4.0 and Internet of Things promise innovative business models and novel user experiences through strong connectivity and effective use of next generation of embedded devices [6].

Industrial and IP-enabled low-power wireless networking technologies are converging, resulting in the IIoT. On the one hand, low-power wireless solutions are available today that answer the strict reliability and power consumption requirements of industrial applications. On the other hand, a range of standards have been published to allow low-power wireless devices to communicate using the Internet Protocol (IP) [7].

Cyber-physical systems are the indispensable technological link for the merging of real and virtual worlds. Cyber-physical systems consist of objects featuring embedded software and electronics that are connected with each other or via the Internet to form a single networked system. They include sensors and components for moving or controlling a mechanism or system, so called actuators linking the CPS to the outside world. The sensors enable the systems to acquire and process data. The data is subsequently made available to network-based services that use actuators to directly impact measures taken in the real world (Fig. 1). This leads to the merging of the physical world and cyberspace into the Internet of Things [8].

The integration of shop floor devices into IIoT platforms, with the concept of Smart-Object (SO), which will enable processing of data to aid decisionmaking, not only with the information of one device, but also with several devices, forming a cyberphysical system.

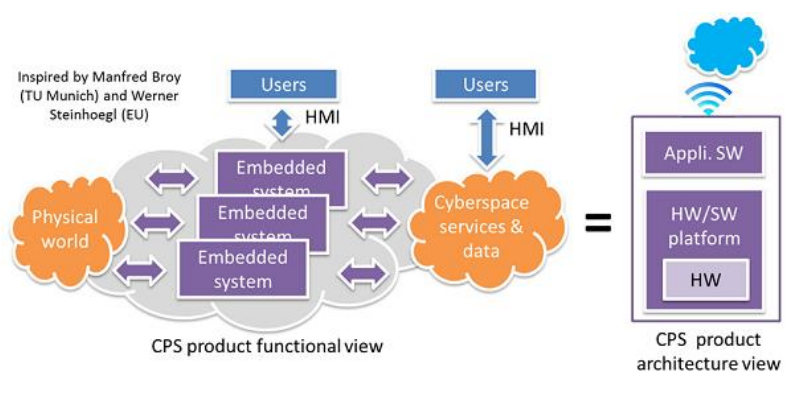

Figure 1: Architecture of a CPS [9].

CPSs are used in industrial production to build up Internet-based architectures that facilitate remote control of stand-alone production systems. This system of sub-systems is composed of application software running on a hardware/software platform, including specific hardware components (Fig. 2).

\section{OPC Servers}

OPC is a standard interface between numerous data sources, - such as programmable logic controllers (PLCs), remote terminal units (RTUs), and sensors on a factory floor - to HMI/SCADA applications, application tools, and databases. With OPC, deviceside server and application software can communicate without duplicating device driver development and providing support for hardware feature changes [10].

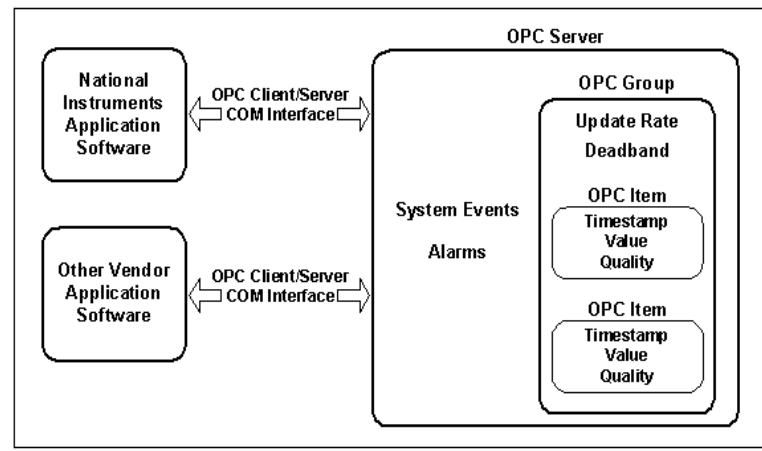

Figure 2: An example implementation of the OPC specification [11].

OPC servers provide a method for many different software packages to access data from a process control device. We have an OPC Server and one or more OPC Clients that communicate with the server to write or read data. In this document, we will use MatrikonOPC Server.

\subsection{Motrikon OPC Server}

MatrikonOPC Simulation Server is a free utility used to help test and troubleshoot OPC applications (clients) and connections. Testing applications on "live" OPC servers may result in loss of actual production data. The MatrikonOPC Simulation Server creates a simulated environment so that in the event of a problem, no real process data is lost.

\section{Industrial Communications}

Industrial communications are at the heart of automated systems. Numerous defined standards exist (RS-485, AS-I, PROFIBUS, PROFINET), and these standards continue to grow as new industrial Ethernet protocols emerge [12].

The vertical integration of management execution systems with factory floor equipment has led to the convergence of the Ethernet TCP/IP protocol with industrial field busses. Several industrial Ethernet protocols have emerged, including Profinet, Ethernet/IP, ModbusTCP/IP, EtherCAT®, and Ethernet Powerlink. Each protocol addresses very specific needs, so requirements on the hardware platform vary accordingly. 


\subsection{RS-485}

RS-485 is a standard defining the electrical characteristics of drivers and receivers for use in serial communications systems. RS-485 allows multiple devices (up to 32) to communicate at halfduplex on a single pair of wires, plus a ground wire (more on that later), at distances up to 1200 meters (Fig. 3). Data is transmitted differentially on two wires twisted together, referred to as a "twisted pair." The use of a balanced line means RS485 has excellent noise rejection and is ideal for industrial and commercial applications [13].

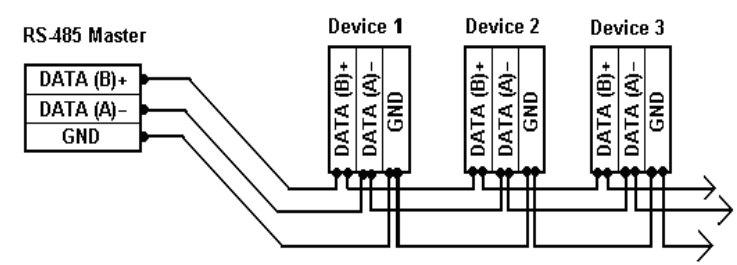

Figure 3: Wire RS-485 connections [13].

\subsection{EtherCAT®}

EtherCAT $\AA$ (Ethernet for Control Automation Technology) is an open industrial real-time and deterministic Ethernet Fieldbus standard that is widely regarded as the best industrial network to date [14]. EtherCAT $\AA$ is based on the principle of 'Ethernet on the fly' processing, a driving factor for its incredibly fast cycle times for industrial automation applications. It supports a wide variety of standard application layer implementations, including: CANopen over EtherCAT (CoE), Ethernet over EtherCAT (EoE), File-access over EtherCAT (FoE), Vendor-specific over EtherCAT (VoE) and Servo Profile over EtherCAT (SoE) mailbox protocols. These previous communication profiles allow multiple vendors to make fully compliant devices with the same application implementations [15].

- CANopen over EtherCAT (CoE): This protocol is used to configure slaves and exchange data objects on application level.

- Ethernet over EtherCAT (EoE): The EtherCAT master implements the Ethernet over EtherCAT mailbox protocol to enable the tunnelling of Ethernet frames to special slaves, that can either have physical Ethernet ports to forward the frames to, or have an own IP stack to receive the frames.

The process management level has special communication requirements that differ slightly from the requirements addressed by the EtherCAT Device Protocol, described previously. Machines or sections of a machine often need to exchange status information and information about the following manufacturing steps with each other. Additionally, there is usually a central controller that monitors the entire manufacturing process, which provides the user with status information on productivity, and assigns orders to the various machine stations. The EtherCAT Automation Protocol (EAP) defines interfaces and services for:

- Exchanging data between EtherCAT master devices (master-master communication)

- Communication to Human Machine Interfaces (HMI)

- A supervising controller to access devices belonging to underlying EtherCAT segments (Routing)

- Integration of tools for the machine or plant configuration, as well as for device configuration.

Specifically, in this chapter, we focus on EtherCAT® industrial protocol with Arduino [15]. The union of the world Arduino and the world EtherCAT $\AA$ will allow you to create innumerable devices for the automation in a simple, rapid and economic way. Some of the possible applications are:

- I/O devices both analogical and digital with possibility of pre-elaboration and elaboration of the signals as averages, linearization, filters, among other functions.

- Generators of PWM signals to pilot motor and other.

- Serial gateways RS-232, RS-485, CAN, Modbus TCP/IP, from and toward EtherCAT®.

\section{Case Study - Predictive Maintenance in Induction Motors based on Vibrations Analysis}

One of the important concepts related to smart devices is the ability to implement machine learning techniques, automatically identify machine anomalies or predict future events. In this work, the classification of vibration gravity was implemented to predict anomalies in electric inductive motors.

This event identification and classification automatically assists maintenance technicians and engineers to predict future problems and act in advance.

Machine learning is a kind of artificial intelligence that provides systems with the ability to learn without being explicitly programmed. Machine learning focuses on developing computer programs that can be changed, when exposed to new data. The algorithms use data to detect patterns and adjust program actions accordingly. Typically, these algorithms can be categorized as being supervised, unsupervised and reinforcement learning. Supervised algorithms can apply what has been learnt in the past to new data. Unsupervised algorithms can draw inferences from datasets. In reinforcement learning, the algorithm learns a policy 
of how to act given an observation of the world. Every action has some impact in the environment, and the environment provides feedback that guides the learning algorithm [16].

Supervised learning includes two categories of algorithms, classification for categorical response values, where the data can be separated into specific "classes" and regression for continuous-response values.

To measure misalignments and eccentricities on the motor shaft, accelerometers were installed on the different axes as shown in Fig. 4.

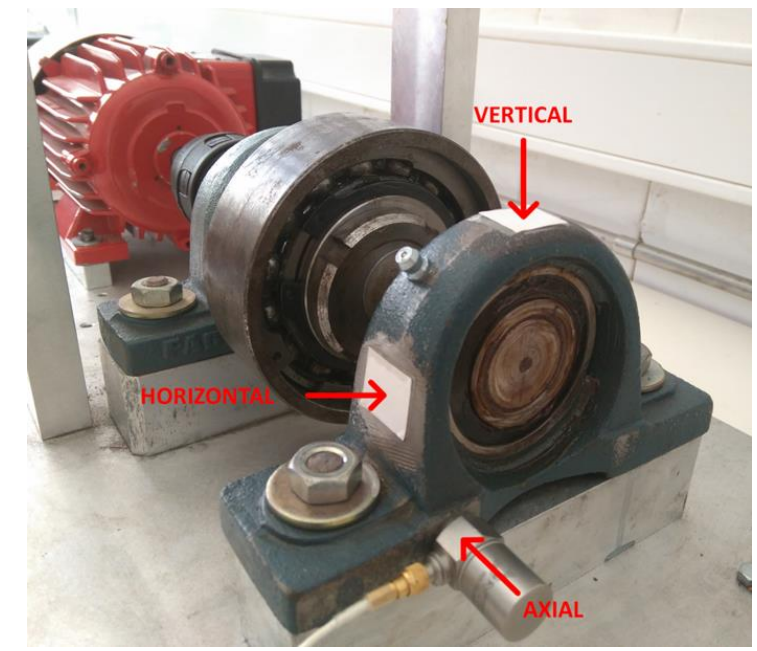

Figure 4: Induction motor coupled to a misalignment setup.

The acquisition and storage of data were made by two different experimental configurations. The first is based on a National Instruments acquisition board and the second based on an Arduino board with an EtherCAT Shield, according to Fig. 5.
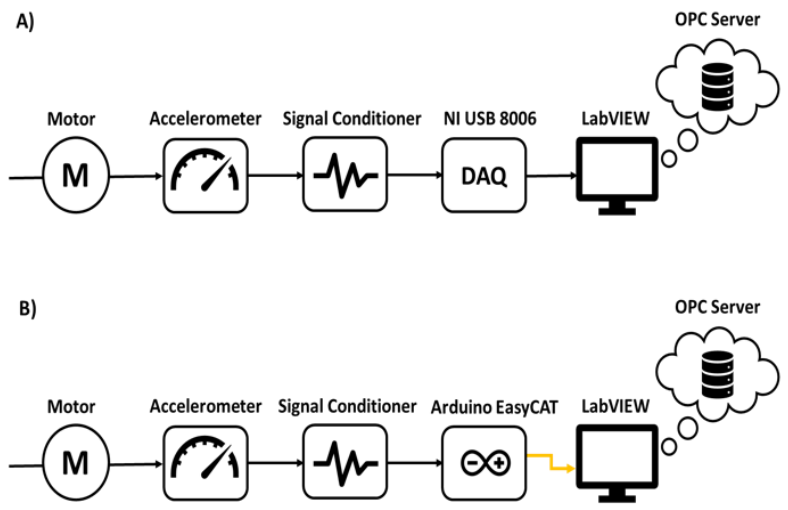

Figure 5: Layout of experimental setup.

A supervised learning model was developed for classification data based on the vibration measurements according to Fig. 6. The vibration's motor severity was classified through the k-nearest neighbour's algorithm (k-NN). The standard ISO 2372 was used in the k-NN supervised learning procedures. This standard indicates a suggested classification, according to vibration's severity range limits (velocity) and machines classes, in four groups: $\mathrm{A}=$ Good, $\mathrm{B}=$ Satisfactory, $\mathrm{C}=$ Unsatisfactory and $\mathrm{D}=$ Unacceptable.

The classification model is trained considering 4000 samples cases, with evident occurrences of the four classes. Before classification, we need to identify the events. For the example of vertical vibration, the system detects an upper peak of vibration velocity, depicted in Fig. 7. Based on the magnitude of the peaks, the system can automatically classify the vibrations. In this way, it is possible to classify vibration's severity in one of these four groups. As the value of the peak is $3,9 \mathrm{~mm} / \mathrm{s}$ and the class of motor is Class I ( $<20 \mathrm{HP})$, the vibration's severity is the Class $\mathrm{C}$, that is, the vibrations are 'Unsatisfactory' -according to ISO 2372 such as it was indicated in the previous data analysis.

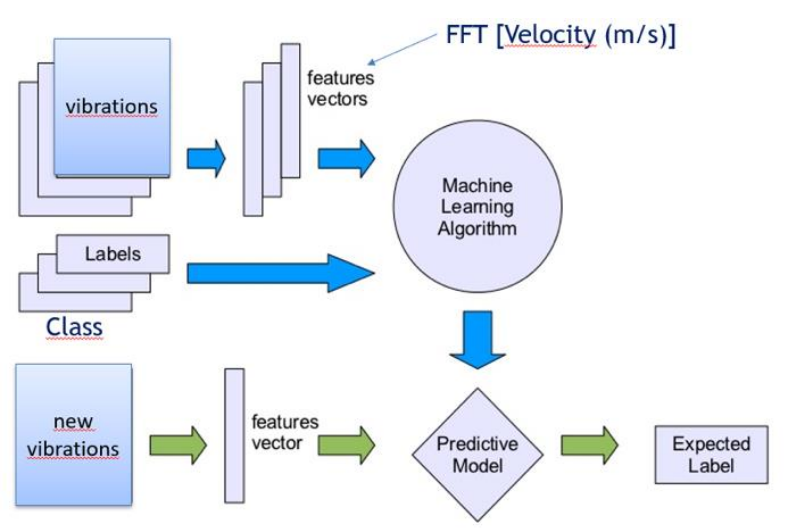

Figure 6: Supervised learning model implemented.

\section{Conclusions}

This paper gives an overview of the technologies currently available to implement a CPS, describing the architecture, communication protocols and information servers. In the case study, the CPS analyse and record the vibration data of an electrical motor to identify the vibration's severity and to implement a predictive maintenance using classifiers according to machine learning fundamentals.

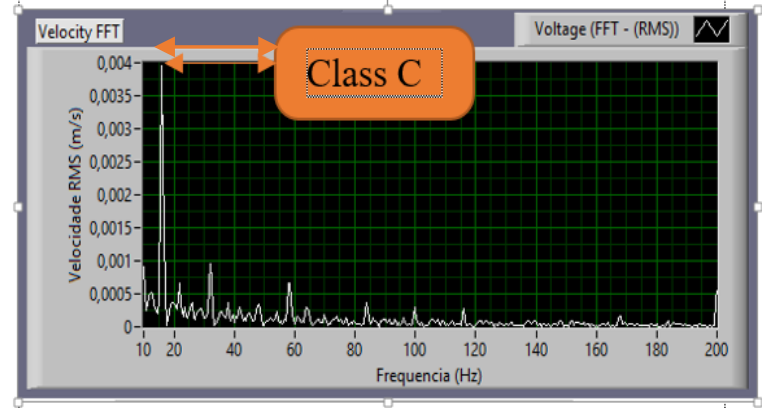

Figure 7: User interface: Velocity's FFT, identification and classification result.

Two systems were implemented for acquisition and analysis of vibration data in induction motors. 
Each system can be understood as a smartbox, capable of serving as a gateway between shop floor equipment and supervisory systems. The smartbox incorporates Machine Learning techniques to classify vibration severity according to ISO-2372. The smartbox can be used for predictive maintenance situations, as well as any connection between supervision and machines in the context of cyber-physical systems.

The proposed implementation successfully detected the vibration severity fault and allowed to demonstrate the concept of Cyber-Physical systems.

Further developments of this work will include the deployment and testing of CPSs using the proposed techniques in real industrial environments, with harsh environmental conditions and a diversity of production machines in the shop floor.

\section{Acknowledgements}

This research was supported by project PRODUTECH-SIF (COMPETE2020).

\section{References}

[1] Y. Liu, Y. Peng, B. Wang, S. Yao, and Z. Liu. Review on cyber-physical systems. IEEE/CAA Journal of Automatica Sinica, 4(1):27-40, Jan 2017.

[2] Atzori, L., Lera, A., Morabito, G.: The Internet of Things: A survey. Computer Networks, pp 1- 19 (2010).

[3] R. Silva, J. Reis, L. Neto, and G. Gonçalves. Universal parser for wireless sensor networks in industrial cyber physical production systems. In 2017 IEEE 15th International Conference on Industrial Informatics (INDIN), pages 633-638, July 2017.
[4] Gubbi, J., Buyya, R., Marusic, S., Palaniswami, M.: Internet of Things (IoT): A Vision, Architectural Elements, and Future Directions. pp. 1-19 (2013).

[5] Da Xu, L., He, W., Li, S.: Internet of Things in Industries: A Survey. IEEE transactions on industrial informatics, vol. 10, no. 4 (2014).

[6] Sadeghi, A., Wachsmann, C., Waidner, M.: Security and Privacy Challenges in Industrial Internet of Things. IEEE Design Automation Conference, pp. 1-6 (2015).

[7] Dujovne, D., Watteyne, T., Vilajosa, X., Thubert, P.: Deterministic IP-Enabled industrial internet (of things). In: IEEE Communications Magazine (2015).

[8] Cyber physical system, https://www.hbm.com, last accessed 2018/01/21.

[9] Architecture of a Cyber physical system, www.eurocps.org, last accessed 2018/01/21.

[10] OPC servers: http://home.hit.no, last accessed 2018/01/21.

[11] OPC specification, http://ni.com, last accessed 2018/01/21

[12] Industrial http://www atmel com 2018/01/21.

[13] RS-485 protocol, www.bb-elec.com, last accessed 2018/01/21.

[14] EtherCAT, https://www.beckhoff.com, last accessed 2018/01/21.

[15] EtherCAT and Arduino, http://www.bausano.net, last accessed 2017/10/2.

[16] Safavian, S. R., Landgrebe, D.: A survey of decision tree classifier methodology. In: IEEE transactions on systems, man, and cybernetics, 21(3), pp. 660-674, (1991).

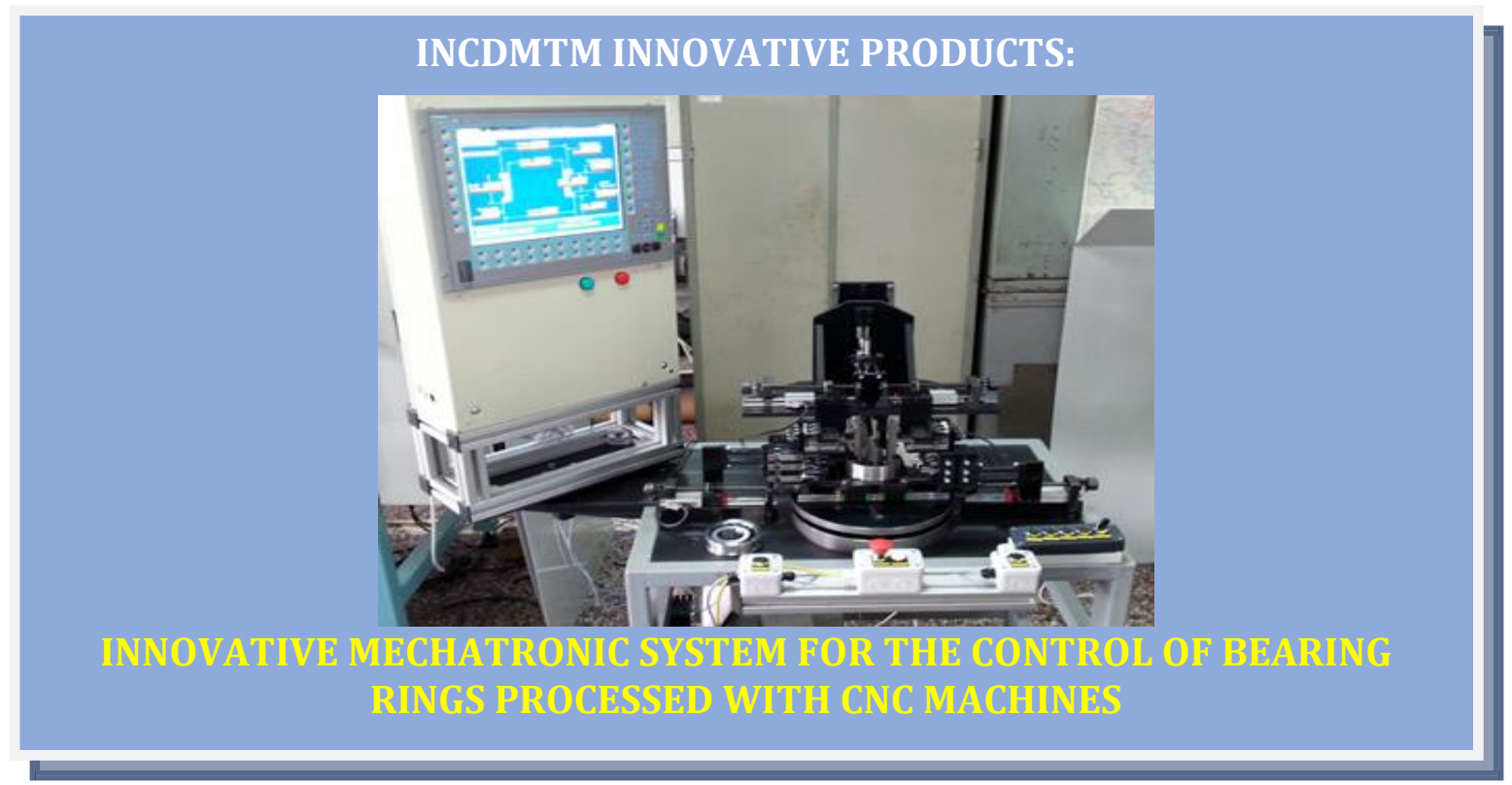

